\title{
Ultra-wideband RCS Reduction and Gain Enhancement of Aperture-Coupled Antenna Based on Hybrid-FSS
}

\author{
Lili CONG, Xiangyu CAO, Tao SONG \\ Information and Navigation College, Air Force Engineering University, Xi' an 710077, China \\ 1183068955@qq.com, xiangyucaokdy@163.com,475559422@qq.com \\ Submitted March 9, 2017 / Accepted July 15, 2017
}

\begin{abstract}
A novel design of aperture-coupled microstrip antenna with ultra-wideband low radar cross section (RCS) is proposed. Hybrid frequency selective surface (FSS) structures consisting of two kinds of polarizationdependent folded split ring resonators (PDFSRRs) and square patches are utilized to replace the conventional metallic ground. By orthogonally arranging the PDFSRRs in a chessboard-like configuration, the band-stop characteristic contributes to the gain enhancement, while the zero degree reflection phase points and wave-transmission characteristic are utilized to achieve RCS reduction both in-band and out-of-band. Furthermore, with square patches periodically etched on the bottom of FSS structures, a new zero degree reflection phase is introduced to enhance the effect of RCS reduction. Full wave simulations and measurements demonstrate that the proposed antenna achieves RCS reduction from $1 \mathrm{GHz}$ to $18 \mathrm{GHz}$ and gain enhancement compared with traditional microstrip antenna.
\end{abstract}

\section{Keywords}

Ultra-wideband, frequency selective surface, polarization dependent, radar cross section, split ring resonator

\section{Introduction}

With the rapid development of stealth and detection technology, antenna radar cross section reduction (RCSR), which contributes significantly to the total RCS of airborne platforms, has sparked an increasingly growing concern. So far many methods have been brought up to reduce antenna RCS, such as geometrical shaping [1], [2] and radar absorbing material (RAM) [3-8]. In the former case, the antenna can deflect the electromagnetic (EM) waves away from the detective direction, thus reducing RCS in specular directions. However, it would inevitably give rise to reflection in some other directions. In the latter case, the RAM would transform the incident wave energy into heat, consequently achieving RCSR. Nevertheless, the degradation of radiation performance would be inevitable as a result. Consequently, it is a severe challenge to reduce RCS without degrading the radiation performance, let alone achieving in-band and out-of-band RCSR and high gain simultaneously. So this paper is presented to provide a feasible approach to address the problem.

Frequency selective surface (FSS), as a typical artificial composite structure, has been extensively applied in antenna RCSR [9-16]. In [17], a Fabry-Perot cavity antenna based on partially reflecting surface (PRS) technology is proposed. It can achieve RCSR both in and out of the operation frequencies while maintaining a high gain. However, the profile of the antenna resulting from the loading height of the PRS superstrate limits its application. Meanwhile, the antenna performance is sensitive to the loading height, which increases fabrication flexibility. In [18], a low profile mushroom antenna with wideband low RCS is proposed by combing mushroom cells, FSS and artificial magnetic conductor (AMC) in a shared aperture. However, the narrow in-phase reflection bandwidth of AMC restricts the RCSR frequency range, and the in-band RCSR is not well designed.

In this paper, hybrid-FSS structures consisting of two kinds of polarization-dependent folded split ring resonators (PDFSRRs) and periodic square patches are proposed and utilized to achieve ultra-wideband RCSR. With the two kinds of PDFSRRs orthogonally arranged around the ground plane, gain enhancement is achieved due to bandstop characteristic, while ultra-wideband RCSR for in-band and out-of-band is achieved because of the introduction of zero degree reflection phase points and wave-transmission characteristic. The periodic square patches are utilized to introduce another new zero degree reflection phase point to further reduce antenna RCS in the high frequency band. Thus, ultra-wideband RCSR and gain enhancement can be achieved in one single step, which makes the whole antenna structure simple and easy to be manufactured. The prototype is fabricated and measured. The results show that compared with [18] the proposed antenna RCS is reduced in an ultra-wideband extending from $1 \mathrm{GHz}$ to $18 \mathrm{GHz}$ with maximum gain enhancement of $2.4 \mathrm{dBi}$. The in-band and out-of-band RCSR can reach up to $5 \mathrm{~dB}$ and $32 \mathrm{~dB}$, respectively. 


\section{Design Principles}

The total RCS of an antenna can be described as [19]:

$$
\sigma=\left|\sqrt{\sigma^{\mathrm{st}}}+\sqrt{\sigma^{\mathrm{an}}} \exp (\mathrm{j} \varphi)\right|^{2}
$$

where $\sigma^{\text {st }}$ represents the structural mode RCS, dependent on the structural characteristics of an antenna such as the metal surface, corners, edges, etc. $\sigma^{\text {an }}$ represents the antenna mode RCS, mainly determined by antenna radiation characteristics and port termination such as load impedance. $\varphi$ is the phase difference between the two components.

For normal incidence, the antenna mode RCS can be defined as [20]:

$$
\sigma_{\mathrm{M}}^{\mathrm{an}}=G^{2} \mu^{2} \Gamma^{2} \lambda^{2} / 4 \pi
$$

where $G$ is the antenna gain, $\lambda$ is the wavelength, $\Gamma$ is the reflection coefficient and $\mu$ is polarization matching between and among the transmitter, antenna and receiver.

Since $\sigma^{\text {an }}$ is highly related to the radiation performance, $\sigma^{\text {an }}$ should remain unchanged in the working frequency range to guarantee highly-effective radiation of EM waves. In the operation frequency band of the proposed antenna, the PDFSRRs exhibit nearly-full-reflection frequency response just like a perfect electric conductor so as to maintain the antenna radiation performance. Meanwhile, the PDFSRRs present band-pass characteristic out of the operation band in such a way that $\sigma^{\text {st }}$ can be reduced to achieve low RCS out of band.

To clearly interpret the mechanism of in- and out-ofband RCSR, a simplest metamaterial (MTM) array consisting of $2 \times 2$ elements is depicted in Fig. 1(a) and the total scattering field can be described as [14]:

$$
\boldsymbol{E}=\boldsymbol{E}_{\mathrm{MTM} 1} \cdot A F_{\mathrm{MTM} 1}+\boldsymbol{E}_{\mathrm{MTM} 2} \cdot A F_{\mathrm{MTM} 2}
$$

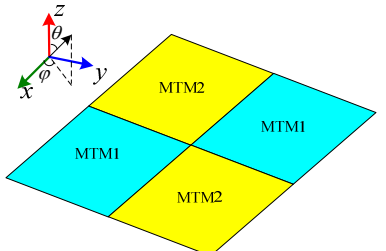

(a)

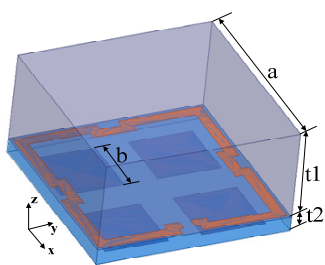

(c)

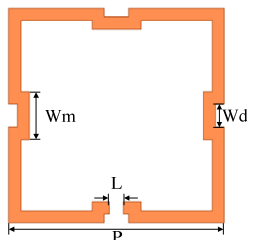

(b)

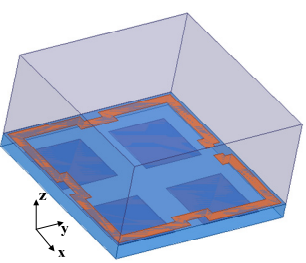

(d)
Fig. 1. (a) Schematic of a $2 \times 2$ MTM array; (b) geometry of the PDFSRR; (c) FSS1 structure; (d) FSS2 structure. The corresponding geometrical parameters are designed as: $\mathrm{a}=6.4 \mathrm{~mm}, \mathrm{~b}=2.1 \mathrm{~mm}, \mathrm{p}=5.5 \mathrm{~mm}$, $\mathrm{L}=0.6 \mathrm{~mm}, \mathrm{Wm}=1.4 \mathrm{~mm}, \mathrm{Wd}=0.7 \mathrm{~mm}, \mathrm{t} 1=3 \mathrm{~mm}$, $\mathrm{t} 2=0.5 \mathrm{~mm}$. where $\boldsymbol{E}$ and $A F$ represent the scattered pattern and array factor respectively.

$$
\begin{gathered}
\boldsymbol{E}_{\mathrm{MTM} 1}=E_{1} \exp \left(\mathrm{j} \varphi_{1}\right) \boldsymbol{E}_{\text {element } 1} \\
\boldsymbol{E}_{\mathrm{MTM} 2}=E_{2} \exp \left(\mathrm{j} \varphi_{2}\right) \boldsymbol{E}_{\text {element2 }}, \\
A F_{\mathrm{MTM} 1}=\exp [\mathrm{j}(k x+k y) d / 2]+\exp [\mathrm{j}(-k x-k y) d / 2], \\
A F_{\mathrm{MTM} 2}=\exp [\mathrm{j}(-k x+k y) d / 2]+\exp [\mathrm{j}(k x-k y) d / 2]
\end{gathered}
$$

where $E_{1}$ and $E_{2}$ are the amplitude of scattering field, $\boldsymbol{E}_{\text {element1 }}$ and $\boldsymbol{E}_{\text {element2 }}$ are polarized direction, $\varphi_{1}$ and $\varphi_{2}$ are phase shift, $d$ is the distance between adjacent elements, $k x=2 \pi \sin \theta \cos \varphi / \lambda, k y=2 \pi \sin \theta \sin \varphi / \lambda$.

For monostatic RCS with incident wave vertically impinging on the MTM array, the scattered electronic field would be the same with the incidence one, and then $\boldsymbol{E}_{\text {element1 }}=\boldsymbol{E}_{\text {element2. Assuming that each element totally }}$ reflects the incidence wave with no absorption, then $E_{1}$ would be as same as $E_{2}$. For normal incidence with $(\theta, \varphi)=(0,0)$, there will be $A F_{\mathrm{MTM} 1}=A F_{\mathrm{MTM} 2}=2$. Thus the total scattering field can be simplified as

$$
\boldsymbol{E}=2 E_{1}\left[\exp \left(\mathrm{j} \varphi_{1}\right)+\exp \left(\mathrm{j} \varphi_{2}\right)\right] \boldsymbol{E}_{\text {element1 }} .
$$

As can be seen the total scattering field is closely related to the phase difference between $\varphi_{1}$ and $\varphi_{2}$. And it can be concluded that once the phase difference between $\varphi_{1}$ and $\varphi_{2}$ varies in the range from $90^{\circ}$ to $270^{\circ}$, the scattering field can be canceled in the backward direction compared with a perfect electric conductor. Hence, the antenna mode RCSR is achieved.

\section{Simulation and Discussion}

\subsection{Design and Analysis of the Unite Cell}

The unite cell of the proposed FSS1 structure is composed of two metallic layers separated by an epoxy FR4 substrate with a dielectric constant of 4.4 and a loss tangent of 0.02, as shown in Fig. 1(c). The top metallic layer is PDFSRR structure and the bottom layer is four square patches with exactly same size. By rotating FSS1 structure $90^{\circ}$ counter-clockwise around z-axis, we can get the unite cell of FSS2 structure as depicted in Fig. 1(d).

The operating frequency of the reference antenna is set to be from $5.17 \mathrm{GHz}$ to $5.76 \mathrm{GHz}$. We use Ansoft HFSS v.14.0 to perform full-wave simulations. Figure 2 depicts the reflection and transmission coefficients of the FSS1 and FSS2 structures. It can be seen that the stop band from $4.5 \mathrm{GHz}$ to $6.1 \mathrm{GHz}$ of FSS1 with TM-polarized incident wave is the same with that of FSS2 with TE-polarized incidence. Both the FSS1 and FSS2 present nearly-full reflection over the operation frequency band. Consequently, the FSS1 and FSS2 can be combined to maintain effective radiation performance. 


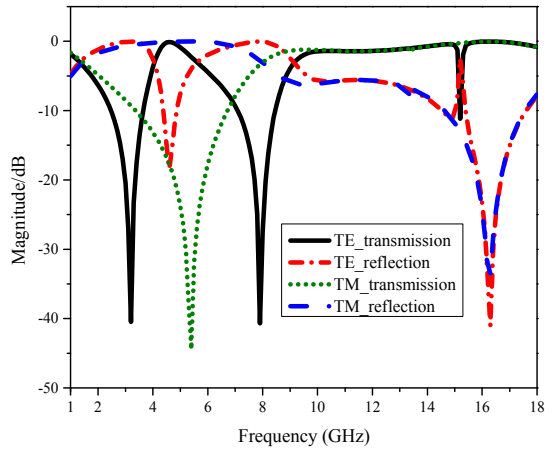

(a)

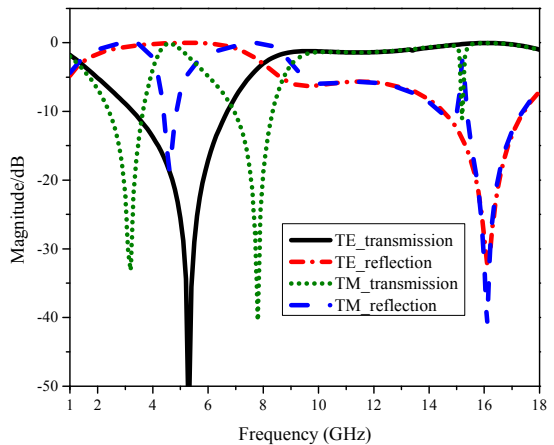

(b)

Fig. 2. Reflection and transmission coefficients: (a) FSS1 structure; (b) FSS2 structure.

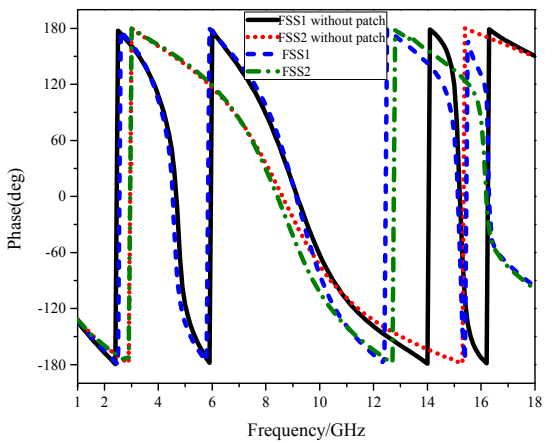

(a)

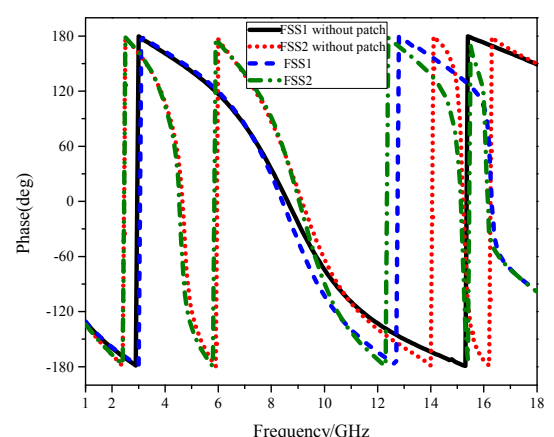

(b)

Fig. 3. Reflection phases of the FSS structures: (a) TE polarization; (b) TM polarization.

Figure 3 shows the reflection phase of FSS1 and FSS2 with and without square patches, respectively. For the case of FSS1 with no patches, when the incidence is TE polarized with electric field perpendicular to the PDFSRR gap, there are three zero points of the reflection phase at 4.66 GHz, $9.13 \mathrm{GHz}$ and $15.20 \mathrm{GHz}$, respectively. In the

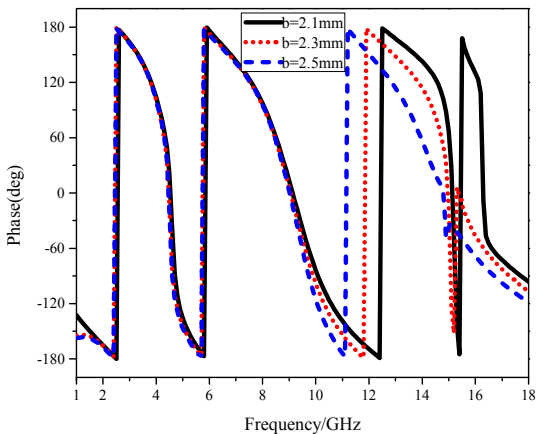

(a)

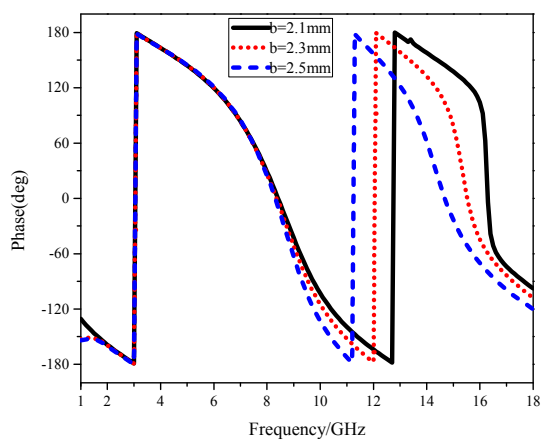

(b)

Fig. 4. Effects on the reflection phase by tuning b: (a) TE polarization; (b) TM polarization.

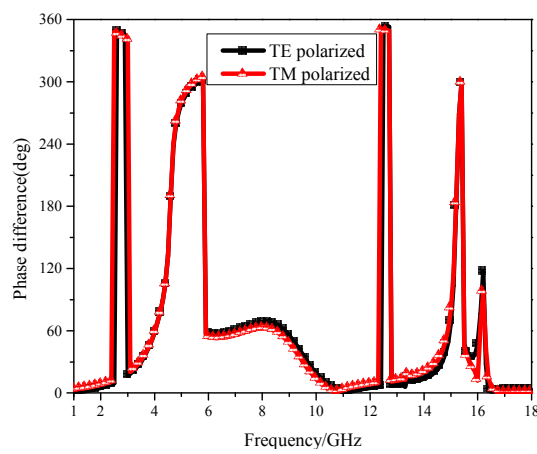

Fig. 5. Reflection phase differences between FSS1 and FSS2.

other hand, there is only one zero reflection phase point at $8.6 \mathrm{GHz}$ when the incidence is TM polarized with electric field parallel to the PDFSRR gap. Similarly, for the case of FSS2 without patches, there is only one zero reflection phase point at $8.6 \mathrm{GHz}$ with TE-polarized incidence, while there are three zero points at $4.65 \mathrm{GHz}, 9.10 \mathrm{GHz}$ and 15.22 GHz with TM-polarized incidence. Thus, we can infer that the combination of these two kinds of PDFSRRs can reduce the in-band and out-of-band RCS for both TE and TM polarizations.

Meanwhile, with square patches etched on the bottom layer of FSS1, the zero points of reflection phase for TE polarization are added to four at $4.54 \mathrm{GHz}, 9.13 \mathrm{GHz}$, $15.14 \mathrm{GHz}$ and $16.3 \mathrm{GHz}$, while two zero points are created at $8.4 \mathrm{GHz}$ and $16.3 \mathrm{GHz}$ for TM polarization. The case of FSS2 is similar to that of FSS1. Consequently, we can infer that the utilization of square patches would strengthen the effect of RCSR. Figure 4 shows that along 
with $b$ increase, the new zero point of reflection phase shifts to lower frequency, while the other reflection phases remain nearly unchanged for both TE and TM polarizations. It can be explained that the narrower gap between adjacent patches leads to $\mathrm{C}$ increase. From above analysis we can conclude that the reflection phase in the low and

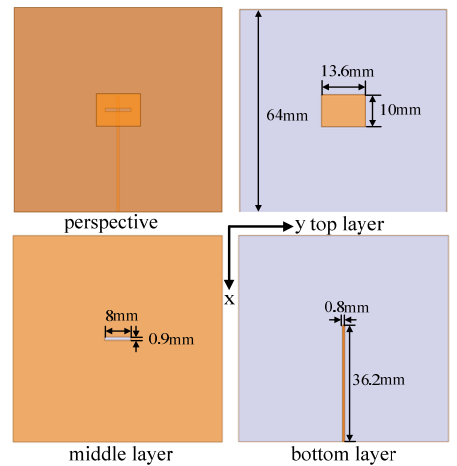

(a)

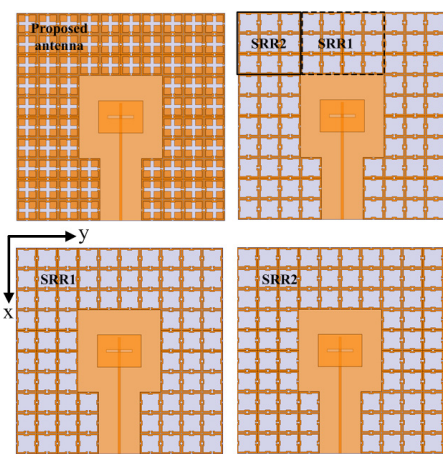

(b)

Fig. 6. (a) The schematic view and geometry of reference antenna; (b) four kinds of arrangement of PDFSRRs and square patches.

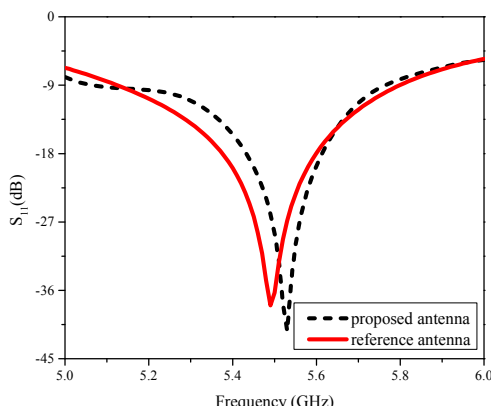

(a)

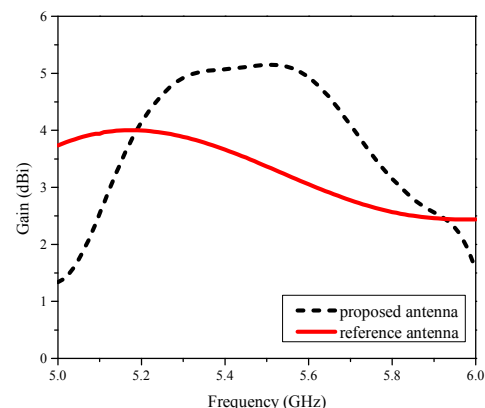

(b)

Fig. 7. Radiation performances comparison: (a) $\mathrm{S}_{11}$; (b) gain. high frequency band can be adjusted independently, which facilitates the procedure of finding the optimal phase difference for phase cancelation.

The reflection phase difference between FSS1 and FSS2 is also investigated. As clearly shown in Fig. 5, the reflection phase difference between FSS1 and FSS2 remains in the range from $90^{\circ}$ to $270^{\circ}$ around $5 \mathrm{GHz}$ and $15 \mathrm{GHz}$. According to the phase cancellation principle [12], [17], the reflection waves from FSS1 and FSS2 will be canceled out in the backward direction for both in- and outof-band.

\subsection{Analysis of the Proposed Antenna}

We use a traditional aperture-coupled microstrip antenna resonant at $5.49 \mathrm{GHz}$ as the reference antenna, as depicted in Fig. 6(a). The reference antenna is fed by a microstrip line through a slot cut in the ground plane. The hybrid FSSs and metal plane is etched on a 3-mm-thick substrate to replace the traditional metallic ground of the reference antenna. The two kinds of PDFSRRs are orthogonally arranged with respect to its neighbor in a chessboard-like configuration. The square patches and the microstrip line is printed on a 0.5 -mm-thick substrate. Both the substrates are with a relative permittivity of 4.4 and a dielectric loss tangent of 0.02 . The metal plane is kept to support the quasi-TEM mode so as to maintain the radiation performance [21].

Figure 7 shows the comparison of $\mathrm{S}_{11}$ and gain. The $\mathrm{S}_{11}$ parameters of the two antennas are nearly the same, while the gain of the proposed antenna is slightly increased

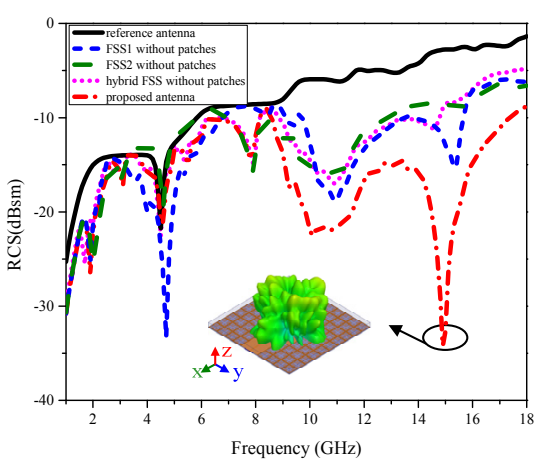

(a)

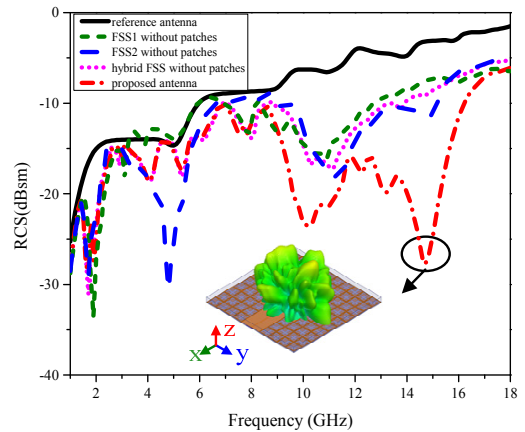

(b)

Fig. 8. RCS versus frequency under normal incidence: (a) TE polarization; (b) TM polarization. 


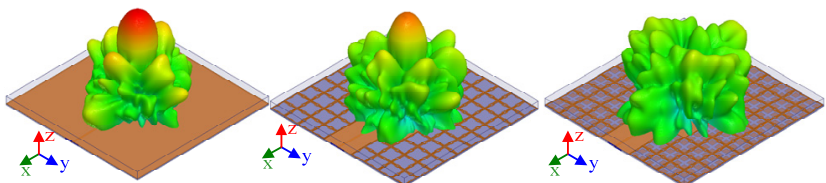

(a)

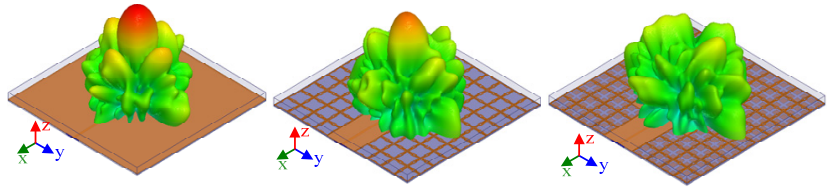

(b)

Fig. 9. The scattering field of the reference antenna and the proposed antenna with and without patches at 14.9 GHz: (a) TE polarization; (b) TM polarization.

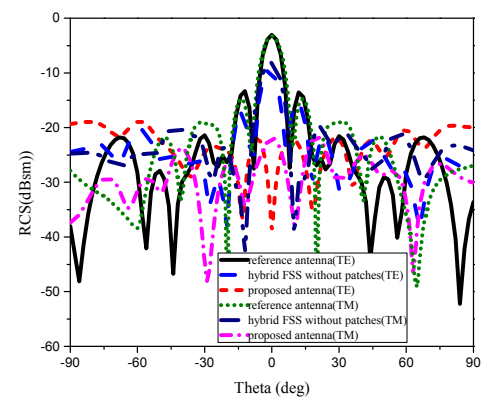

(a)

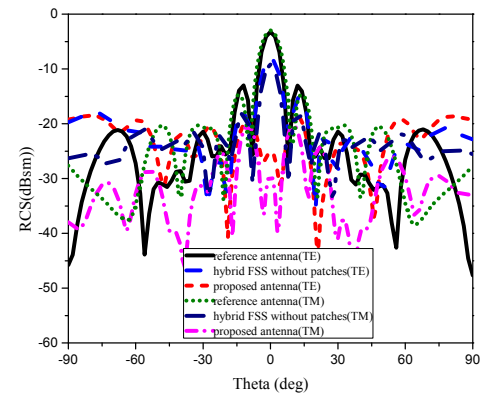

(b)

Fig. 10. RCS versus angle under normal incidence at $14.9 \mathrm{GHz}$ : (a) xoz-plane; (b) yoz-plane.

with a maximum gain enhancement of $2 \mathrm{dBi}$. The simulated results effectively indicate that the radiation performance of the proposed antenna can be well maintained.

To better interpret the effect of the PDFSRRs and square patches on the RCS reduction, we set the other two conditions as comparison as shown in Fig. 6(b). One is to replace the metallic ground of the reference antenna by FSS1 structure without patches, while the other is to replace the metallic ground by FSS2 structure with no patches. For normal incidence, the simulated RCS of the four conditions are depicted in Fig. 8. For the case of FSS1 with no patches, there is a significant RCS reduction around $4.7 \mathrm{GHz}$ for TE polarization, while no RCS reduction is achieved for TM polarization. The reason is that a new zero degree point of the reflection phase at $4.66 \mathrm{GHz}$ is introduced for TE-polarized incident wave in contrast to the case of TM polarization. On the contrary, there is a considerable RCS reduction around $4.8 \mathrm{GHz}$ for TM polarization, while no RCS reduction is achieved for TE polarization for the case of FSS2 without patches. The difference results from the introduction of a new zero reflection phase point at $4.65 \mathrm{GHz}$ for TM polarization. With the utilization of hybrid FSSs without patches, RCSR is achieved around $5.49 \mathrm{GHz}$ (the resonant frequency of the reference antenna) for both TE and TM polarizations. The in-band RCSR can reach up to $4.7 \mathrm{~dB}$ compared with the reference antenna.

In addition, the effect of RCSR for the proposed antenna is observably better than the antenna with no patches. The reason is that the periodic square patches introduce a new zero degree point of reflection phase at 15.2 GHz, which further reduces RCS in the high frequency band. As clearly shown in Fig. 9, the reflective energy is divided into several lobes with the introduction of square patches, and then the RCS is significantly reduced as expected.

The scattering patterns as a function of angle at 14.9 GHz under normal incidence for both TE and TM polarizations are also investigated, as shown in Fig. 10. As a result of phase cancellation and wave transmission, the peak RCSR in xoz-plane and yoz-plane for TE polarization is $35.74 \mathrm{~dB}$ and $16.95 \mathrm{~dB}$, respectively, while it is $13.83 \mathrm{~dB}$ and $20 \mathrm{~dB}$ for TM polarization. Meanwhile, the strong reflection at other directions caused by phase cancellation is suppressed due to the transmission characteristic of hybrid FSSs.

\section{Fabrication and Measurement}

To verify the effectiveness of our design, the prototype was fabricated and measured. Figure 11 shows the photographs of the reference antenna and the proposed antenna. Both the PDFSRRs and square patches are built on a FR4 board through standard wet-etching technology. The $\mathrm{S}_{11}$ parameter was obtained by vector network analyzer Agilent N5230C, as shown in Fig. 12. The measured operating band of the proposed antenna is from $5.14 \mathrm{GHz}$ to $5.8 \mathrm{GHz}$, which agrees well with the simulated one. Figure 13 depicts the comparison of the measured radiation

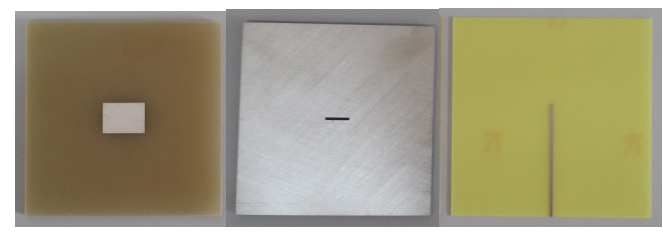

(a)

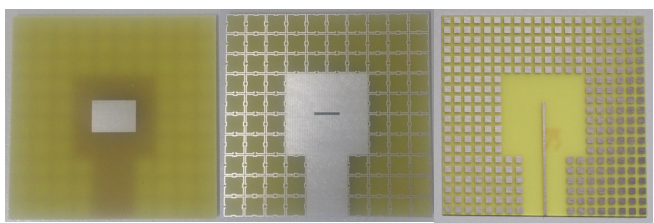

(b)

Fig. 11. Photograph of (a) the reference antenna and (b) the proposed antenna. 


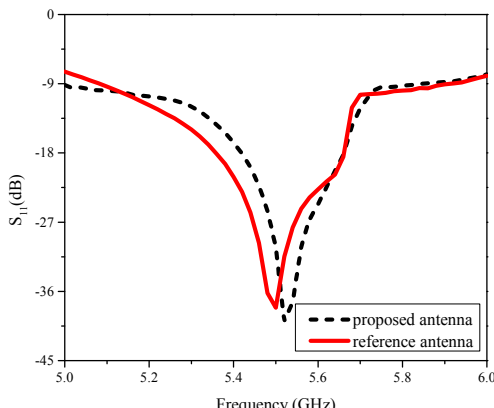

Fig. 12. Measured $\mathrm{S}_{11}$ of the reference and proposed antennas.

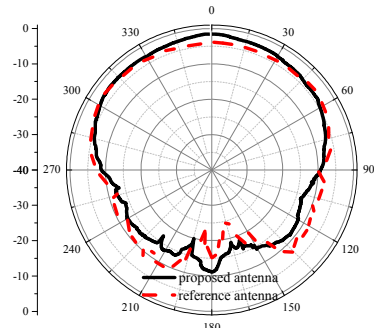

(a)

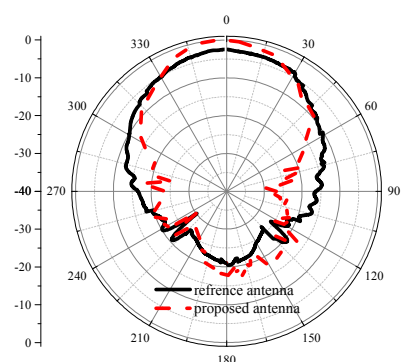

(b)

Fig. 13. Measured gain of the reference and proposed antennas at $5.5 \mathrm{GHz}$ (a) H-plane (xoz-plane); (b) E-plane (yozplane).

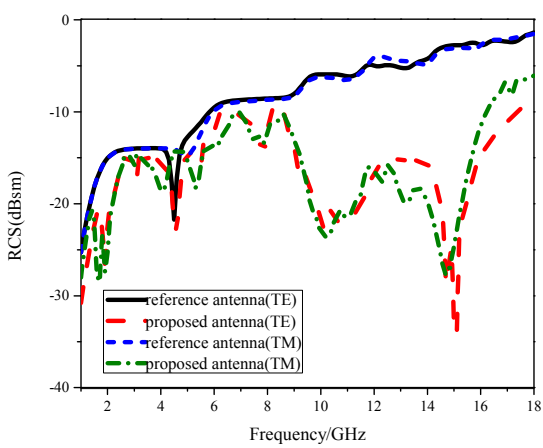

Fig. 14. Measured RCS under normal incidence wave with TE polarization and TM polarization.

patterns at $5.5 \mathrm{GHz}$. The half power beam-width (HPBW) for xoz-plane and yoz-plane is suppressed from $155^{\circ}$ to $108^{\circ}$ and from $110^{\circ}$ to $59^{\circ}$, respectively. The gain of the proposed antenna increases $2.4 \mathrm{~dB}$ compared with that of the reference antenna, which is in good agreement with the simulated one.

To verify the scattering performance of the proposed antenna, the measurement was conducted in an anechonic chamber. Two pyramidal horn antennas are used as trans- mitter and receiver, respectively. The time-domain gating strategy was used to eliminate the undesirable signals. Both antennas under measurement are terminated with a matched load. Figure 14 shows the measured RCS comparison under normal incidence. An ultra-wideband RCSR can be observed from $1 \mathrm{GHz}$ to $18 \mathrm{GHz}$. The in-band RCSR can reach up to $5 \mathrm{~dB}$, while the out-of-band RCSR over $10 \mathrm{~dB}$ is obtained from $1.58 \mathrm{GHz}$ to $2.02 \mathrm{GHz}(24.4 \%$ bandwidth) and from $9.22 \mathrm{GHz}$ to $16.22 \mathrm{GHz}$ (55\% bandwidth). Considering fabrication and measurement errors, satisfactory agreement can be found between measured and simulated results, which confirms the effectiveness of our design.

\section{Conclusion}

A hybrid-FSS composed of PDFSRR and square patches is investigated. By replacing the metallic ground with the hybrid-FSS in a chessboard-like configuration, the proposed antenna achieves gain enhancement and ultrawideband low RCS in-band and out-of-band simultaneously. The proposed design provides a feasible approach to broaden the RCSR bandwidth while achieving gain enhancement. Moreover, it is worth noting that the hybridFSS can be arranged in other configurations and applied to other antennas.

\section{Acknowledgments}

This work is supported by the National Natural Science Foundation of China (No. 61271100, No. 61471389, No. 61671464 and No. 61501494). All authors thank reviewers for their valuable comments.

\section{References}

[1] JIANG, W., LIU, Y., GONG, S. X., HONG, T. Application of bionics in antenna radar cross section reduction. IEEE Antennas and Wireless Propagation Letters, 2009, vol. 8, p. 1275-1278. DOI: 10.1109/LAWP.2009.2037168

[2] THAKARE, Y. B., RAJKUMAR. Design of fractal patch antenna for size and radar cross-section reduction. IET Microwaves, Antennas \& Propagation, 2010, vol. 4, no. 2, p. 175-181. DOI: 10.1049/iet-map.2008.0325

[3] ZHAI, H., ZHAN, C., LI, Z., LIANG, C. A triple-band ultrathin metamaterial absorber with wide-angle and polarization stability IEEE Antennas and Wireless Propagation Letters, 2015, vol. 14, p. 241-244. DOI: 10.1109/LAWP.2014.2361011

[4] FANTE, R. L., MCCORMACK, M. T. Reflection properties of the Salisbury screen. IEEE Transactions on Antennas and Propagation, 1988 , vol. 36 , no. 10, p. 1443-1454. DOI: $10.1109 / 8.8632$

[5] XU, H.-X., WANG, G.-M., QI, M.-Q., et al. Triple-band polarization-insensitive wide-angle ultraminiature metamaterial transmission line absorber. Phyical Review B, 2012, vol. 86, no. 20, p. 205104-(1-10). DOI: 10.1103/PhysRevB.86.205104 
[6] WANG, B. Y., LIU, S. B., BIAN, B. R., et al. A novel ultrathin and broadband microwave metamaterial absorber. Journal of Applied Physics, 2014, vol. 116, no. 9, p. 094504-(1-7). DOI: $10.1063 / 1.4894824$

[7] COSTA, F., MONORCHIO, A. Multiband electromagnetic wave absorber based on reactive impedance ground planes. IET Microwave, Antennas and Propagation, 2010, vol. 4, no. 11, p. 1720-1727. DOI: 10.1049/iet-map.2009.0359

[8] LIU, T., CAO, X. G., GAO, J., et al. RCS reduction of waveguide slot antenna with metamaterial absorber. IEEE Transactions on Antennas and Propagation, 2013, vol. 61, no. 3, p. 1479-1484. DOI: 10.1109/TAP.2012.2231922

[9] TURPlin, J. P., SIEBER, P. E., WERNER, D. H. Absorbing ground planes for reducing planar antenna radar cross-section based on frequency selective surfaces. IEEE Antennas and Wireless Propagation Letters, 2013, vol. 12, p.1456-1459. DOI: 10.1109/LAWP.2013.2288682

[10] JIA, Y. T., LIU, Y., WANG, H., GONG, S. X. Low RCS microstrip antenna using polarization-dependent frequency selective surface. Electronic Letters, 2014, vol. 50, no. 14, p. 978-979. DOI: 10.1049/el.2014.1003

[11] EDALATI, A., SARABANDI, K. Wideband, wide angle, polarization independent RCS reduction using nonabsorptive miniaturized-element frequency selective surfaces. IEEE Transactions on Antennas and Propagation, 2014, vol. 62, no. 2, p. 747-754. ISSN: 0018-926X. DOI: 10.1109/TAP.2013.2291236

[12] PAQUAY, M., IRIARTE, J. C., EDERRA, I., GONZALO, R., DE MAAGT, P. Thin AMC structure for radar cross-section reduction. IEEE Transactions on Antennas and Propagation, 2007, vol. 55, no. 12 , p. 3630-3638. DOI: 10.1109/TAP.2007.910306

[13] IRIARTE GAlARREGUI, J. C., TEllECHEA PEREDA, A., MARTINEZ DE FALCON, J. L. et al. Broadband radar crosssection reduction using AMC technology. IEEE Transactions on Antennas and Propagation, 2013, vol. 61, no. 12, p. 6136-6143. DOI: $10.1109 /$ TAP.2013.2282915

[14] LI, M., XIAO, S., BAI, Y. Y., WANG, B. Z. An ultrathin and broadband radar absorber using resistive FSS. IEEE Antennas and Wireless Propagation Letters, 2012, vol. 11, p. 748-751. DOI: 10.1109/LAWP.2012.220636

[15] XIE, D. P., LIU, X. G.,GUO, H. P., et al. Wideband absorber with multi-resonant gridded-square FSS for antenna RCS reduction. IEEE Antennas and Wireless Propagation Letters, 2016, vol. 16, p. 629-632. DOI: 10.1109/LAWP.2016.2594213

[16] AL-SHEIKH, A., SHEN, Z. X. Design of wideband bandstop frequency-selective structures using stacked parallel strip line arrays. IEEE Transactions on Antennas and Propagation, 2016 vol. 64, no. 8, p. 3401-3409. DOI: 10.1109/TAP.2016.2570247

[17] JIA, Y. T., LIU, Y., WANG, H., et al. Low RCS high-gain and wideband mushroom antenna. IEEE Antennas and Wireless Propagation Letters, 2015, vol. 14, p. 277-280. DOI: 10.1109/LAWP.2014.2363071

[18] PAN, W. B., HUANG, C., CHEN, P., et al. A low-RCS and highgain partially reflecting surface antenna. IEEE Transactions on Antennas and Propagation, 2014, vol. 62, no. 2, p. 945-949. DOI: 10.1109/TAP.2013.2291008

[19] HU, S. M., CHEN, H. H., LAW, C. L., et al. Backscattering cross section of ultrawideband antennas. IEEE Antennas and Wireless
Propagation Letters, 2007, vol. 6, p. 70-73. DOI: 10.1109/LAWP.2007.893069

[20] YANG, S. T., LING, H. RCS of a microstrip leaky-wave antenna. IEEE Antennas and Wireless Propagation Letters, 2013, vol. 12, p. 35-38. DOI: 10.1109/LAWP.2012.2236677

[21] GENOVESI, S., COSTA, F., MONORCHIO, A. Low-profile array with reduced radar cross section by using hybrid frequency selective surfaces. IEEE Transactions on Antennas and Propagation, 2012, vol. 60, no. 5, p. 2327-2335. DOI: 10.1109/TAP.2012.2189701

\section{About the Authors ...}

Lili CONG was born in Weihai Shandong province, P.R. China in 1991. She received B.S. and M.S. from the Air Force Engineering University, Xi'an China, in 2013 and 2015. She is currently working toward Ph.D. degree at the Information and Navigation College of the Air Force Engineering University. Her main interests include metamaterial design and wideband RCS reduction.

Xiangyu CAO received the B.Sc and M.A.Sc degrees from the Air Force Missile Institute in 1986 and 1989, respectively. She joined the Air Force Missile Institute in 1989 as an assistant teacher. She became an associate professor in 1996. She received Ph.D. degree in the Missile Institute of Air Force Engineering University in 1999. From 1999 to 2002, she was engaged in postdoctoral research in Xidian University, China. She was a Senior Research Associate in the Dept. of Electronic Engineering, City University of Hong Kong from June 2002 to Dec 2003. She is currently a professor of the Information and Navigation College, Air Force Engineering University of CPLA. She is the IEEE senior member from 2008. She has authored and coauthored more than 200 technical journal articles and conference papers, and holds one China soft patent. She is the coauthor of two books entitled, Electromagnetic Field and Electromagnetic Wave, and Microwave Technology and Antenna published in 2007 and 2008, respectively. Her research interests include smart antennas, electromagnetic metamaterial and their antenna applications, and electromagnetic compatibility. She is a reviewer of Applied Physics Letter, Journal of Applied Physics, IEEE Transactions on Antennas \& Propagation, and IEEE Antennas Wireless Propagation Letter.

Tao SONG has received B.S. and M.S. from the Air Force Engineering University, Xi' an China, in 2013 and 2015 respectively. He is currently working toward Ph.D. degree at the Air and Missile Defense College, Air Force Engineering University. His research focuses on electromagnetic theory and application. 